\title{
Lie Group for MHD and Reaction Porosity Effects of Variable Viscosity on Heat Generation and Mass Transfer Fluid
}

\section{Abdel-Rahman $\mathrm{GM}^{1 *}$ and Alessa $\mathrm{NA}^{2}$}

${ }^{1}$ Department of Mathematics, Faculty of Science, Benha University, 13518 Benha, Egypt

${ }^{2}$ Department of Mathematical Science, Faculty of Science, Princess Nourah Bint Abdulrahman University, 11671 Riyadh, Saudi Arabia

\begin{abstract}
Magnetohydrodynamic and chemical reaction effects of variable viscosity on heat generation and mass transfer viscous dissipation fluid in the presence of suction/injection through porosity is solved numerically. Lie symmetry group transformations are used to convert the boundary layer equations into non-linear ordinary differential equations. Finally, numerical results are presented for velocity, temperature and concentration profiles for different parameters of the problem are studied. In addition, the effects of the pertinent parameters on the skin friction, the rate of heat transfer and mass fluxes are also discussed numerically.
\end{abstract}

Keywords: Lie group; Thermal reaction; Porous medium; Heat generation; Temperature depended fluid viscosity

\section{Nomenclature}

$A$ : Non-dimensional fluid viscosity of temperature,

$A_{1}$ : Non-dimensional fluid viscosity of concentration,

A: Positive constant,

$B_{0}$ : Uniform magnetic field,

$b$ : Positive constant,

c: Positive constant,

$C_{p}:$ Heat capacity at constant pressure,

$C_{w}$ : surface concentration,

$C_{\infty}$ : Full stream concentration,

$D$ : Diffusional coefficient,

$E_{c}$ : Eckert number $\left(=U_{2} /\left(T_{w}-T_{\infty}\right) c_{p}\right)$,

$g$ : Temperature profile,

$g^{*}$ : Gravity field,

$h$ : Concentration profile,

$L_{e}:$ Liwes number $\left(=v_{0} / D\right)$,

$K$ : Permeability of the porous medium,

$k^{*}$ : Mean absorption coefficient,

$k_{1}$ : Rate of chemical reaction,

$M$ : Magnetic field parameter, $\left(=\sigma B_{0}^{2} / \rho\right)$,

$m$ : Power law exponent, is also constant,

$Q_{1}$ : Volumetric heat source/sink rate,

$P_{r}$ : Prandtl number $\left(=\mu_{0} c_{p} / K\right)$,

$q_{r}$ : Radiative heat flux,

$T$ : Temperature profile,

$T_{w}$ : Wall temperature,

Tळ: Full stream temperature,
$S$ : Porosity parameter $(=01 / \mathrm{k})$,

$R:$ Thermal radiation parameter $(=* \star 2 / 4 \infty \mathrm{k} \mathrm{k} \sigma \mathrm{T})$,

$u$ : Components velocity in the $\mathrm{x}$-direction,

$v$ : Components velocity in the $\mathrm{y}$-direction,

$V$ : Velocity of suction fluid,

$V_{0}$ : Constant velocity

\section{Greek symbols}

$\alpha_{1}$ : Temperature buoyancy parameter $\left(=g^{*} \beta / b v_{0}\right)$,

$\alpha_{2}$ : Concentration buoyancy parameter $\left(=g^{*} \beta^{*} / b v_{0}\right)$,

$\delta_{1}$ : Fluid viscosity of temperature $\left(=A v_{0}\right)$,

$\delta_{2}:$ Positive constant $\left(=a v_{0}\right)$,

$\delta_{3}$ : Fluid viscosity of concentration $\left(=A_{1} v_{0}\right)$,

$\beta$ : Volumetric coefficient of thermal expansion,

$\beta^{*}$ : Volumetric coefficient of concentration expansion,

$\Gamma$ : Heat generation/absorption parameter $\left(=Q_{0} / \mu_{0} c_{p}\right)$,

$\gamma$ : Chemical reaction parameter $\left(=\Omega / v_{0}\right)$,

$\theta$ : Dimensional temperature profile,

$\phi$ : Dimensional concentration profile,

$\eta$ : Similarity variable,

$\lambda$ : Suction/injection parameter $\left(=4 V_{0} / 3 v_{0}\right)$,

*Corresponding author: Abdel-Rahman GM, Department of Mathematics, Faculty of Science, Benha University, 13518 Benha, Egypt, Tel: 0133225494; E-mail: gamalm60@yahoo.com

Received October 24, 2017; Accepted February 20, 2018; Published February 28, 2018

Citation: Abdel-Rahman GM, Alessa NA (2018) Lie Group for MHD and Reaction Porosity Effects of Variable Viscosity on Heat Generation and Mass Transfer Fluid. J Generalized Lie Theory Appl 12: 287. doi: 10.4172/1736-4337.1000287

Copyright: (c) 2018 Abdel-Rahman GM, et al. This is an open-access article distributed under the terms of the Creative Commons Attribution License, which permits unrestricted use, distribution, and reproduction in any medium, provided the original author and source are credited. 
K: Thermal conductivity,

$\mu$ : Viscosity of the fluid,

$\mu_{0}$ : Constant value viscosity of the fluid,

$\rho$ : Density of the fluid,

$v_{0}$ : kinematic viscosity of the fluid $\left(=\mu_{0} / \rho\right)$,

$\sigma$ : Electrical conductivity,

$\sigma^{*}:$ Stephan-Boltzmann constant

\section{Subscripts}

$w, \infty$ : Stand for the wall and free stream conditions

\section{Introduction}

In many engineering processes and geophysical applications such as geothermal reservoirs, drying of porous solids, thermal insulation, enhanced oil recovery, packed bed catalytic reactors and cooling of nuclear reactors. Many practical diffusive operations involve the molecular diffusion of species in the presence of a chemical reaction within or at the boundary layer.

Lie group transformation, also called symmetry analysis, was developed by Sophus Lie to find point transformations which map a given partial differential equation to it. This method has been used by many researchers to solve some nonlinear problems in fluid mechanics [1-3]. Heat transfer in a liquid film on an unsteady stretching surface by Andersson et al. [4], the production of sheeting material arises in a number of industrial manufacturing processes and includes both metal and polymer sheets. It is well known that the flow in a boundary layer separates in the regions of adverse pressure gradient and the occurrence of separation has several undesirable effects in so far as it leads to increase in the drag on the body immersed in the flow and adversely affects the heat transfer from the surface of the body. Several methods have been developed for the purpose of artificial control of flow separation. Separation can be prevented by suction as the lowenergy fluid in the boundary layer is removed $[5,6]$.

Heat transfer in a porous medium over a stretching surface with internal heat generation and suction or injection by Elbashbeshy et al. [7], Mukhopadhyay et al. [8] studied of MHD boundary layer flow over a heated stretching sheet with variable Viscosity, Mukhopadhyay et al. [9] investigated effects of thermal radiation and variable fluid viscosity on free convective flow and heat transfer past a porous stretching surface.

Very recently, Loganathan et al. [10] studied Lie group analysis for the effects of variable fluid viscosity and thermal radiation on free convective heat and mass transfer with variable stream condition. Our aim in this analysis is to consider the effect of the Magneto hydrodynamic and chemical reaction of variable viscosity on heat generation and mass transfer viscous dissipation fluid in the presence of suction/injection through porosity. And the influence various physical parameters on the numerical results will also be discussed. In addition, the effects of the pertinent parameters on the skin friction, the rate of heat transfer and mass fluxes are also discussed.

\section{Formulation of the Problem}

Consider the steady, viscous dissipating laminar flow and heat generation and mass transfer fluid over a vertical stretching sheet emerging out of a slit at origin in the presence chemical reaction through a porous medium and moving with non-uniform velocity $U(x)$ under the influence of a transversely applied magnetic field $\mathrm{B}$ is considered. The temperature dependent fluid viscosity in the form $\mu=\mu_{0}[a+b(T w-T)][11]$. The governing equation for the flow by using incompressible fluid is

Continuity Eq.:

$\frac{\partial u}{\partial x}+\frac{\partial v}{\partial y}=0$

Momentum Eq.:

$u \frac{\partial u}{\partial x}+v \frac{\partial v}{\partial y}=\frac{1}{\rho} \frac{\partial}{\partial y}\left(\mu(T) \frac{\partial u}{\partial y}\right)+g^{*} \beta\left(T-T_{\infty}\right)+g^{*} \beta\left(C-C_{\infty}\right)-\frac{\sigma B^{2}}{\rho} u-\frac{\mu(T)}{\rho K} u$

Energy Eq.:

$u \frac{\partial T}{\partial x}+v \frac{\partial T}{\partial y}=\frac{K}{\rho c_{p}} \frac{\partial^{2} T}{\partial y^{2}}-\frac{1}{\rho c_{p}} \frac{\partial q_{r}}{\partial y}+\frac{Q_{1}}{\rho c_{p}}\left(T-T_{\infty}\right)+\frac{\mu(T)}{\rho c_{p}}\left(\frac{\partial u}{\partial y}\right)^{2}$

Diffusion Eq.:

$u \frac{\partial C}{\partial x}+v \frac{\partial C}{\partial y}=\mathrm{D} \frac{\partial^{2} C}{\partial y^{2}}-k_{1}\left(C-C_{\infty}\right)$

The boundary conditions are:

$u=U(x), v=-V_{0}, T=T_{w}, C=C_{w}$ at $y=0$

Using the Rossel and approximation (Rashed [12]), the radiative heat flux $q_{r}$ is given by

$$
\mathrm{qr}=-\frac{4 \sigma^{*}}{3 k^{*}} \frac{\partial T^{4}}{\partial y}
$$

Assuming that the temperature difference within the flow is sufficiently small such that $T^{4}$ could be approached as the linear function of temperature;

$T^{4} \cong 4 T_{\infty}^{3} T-3 T_{\infty}^{4}$

We introduce the following relations for $u, v, \theta$ and $\phi$ as

$u=\frac{\partial \psi}{\partial y}, v=\frac{\partial \psi}{\partial x}, T=T_{\infty}+\left(T_{w}-T_{\infty}\right) \theta$ and $C=C_{\infty}+\left(C_{w}-C_{\infty}\right) \varphi$

where $\psi$ is the stream function. The stream wise velocity and the suction/injection velocity are:

$$
U(x)=c x^{m}, V(x)=V_{0} x^{\frac{m-1}{2}}
$$

We assumed the form of the magnetic field $B(x)=B_{0} x^{-1 / 4}$ and the permeability of the porous medium $K(x)=k_{0} x^{1 / 2}$.

Eqn. (1) is satisfied automatically and Eqns. (2)-(5) we get:

$$
\begin{aligned}
& \frac{\partial \psi}{\partial y} \frac{\partial^{2} \psi}{\partial x \partial y}-\frac{\partial \psi}{\partial x} \frac{\partial^{2} \psi}{\partial y^{2}}=-A v_{0} \frac{\partial \theta}{\partial y} \frac{\partial^{2} \psi}{\partial y^{2}}+v_{0}[a+A(1-\theta)] \frac{\partial^{3} \psi}{\partial y^{3}}+\frac{g^{*} A \beta}{b} \theta+\frac{g^{*} A_{1} \beta^{*}}{b} \varphi \\
& -x-1 / 2\left[\frac{\sigma B_{0}^{2}}{\rho}+\frac{v_{0}}{k_{0}}(a+A(1-\theta))\right] \frac{\partial \psi}{\partial y} \\
& \frac{\partial \psi}{\partial y} \frac{\partial \theta}{\partial x}-\frac{\partial \psi}{\partial x} \frac{\partial \theta}{\partial y}=\frac{\kappa}{\rho c_{p}} \frac{\partial^{2} \theta}{\partial y^{2}}+\frac{16 \sigma^{*} T_{\infty}^{3}}{3 k^{*} \rho c_{p}} \frac{\partial^{2} \theta}{\partial y^{2}}+\frac{\mathrm{Q} 1}{\rho c_{p}} \theta+\frac{v_{0}}{\left(T_{w}-T_{\infty}\right) c_{p}}[a+A(1-\theta)]\left(\frac{\partial^{3} \psi}{\partial y^{3}}\right)^{2}, \\
& \frac{\partial \psi}{\partial y} \frac{\partial \theta}{\partial x}-\frac{\partial \psi}{\partial x} \frac{\partial \theta}{\partial y}=D \frac{\partial^{2} \varphi}{\partial y^{2}}-k_{1} \varphi .
\end{aligned}
$$

and

$$
\frac{\partial \psi}{\partial y}=c x^{m}, \frac{\partial \psi}{\partial x} V_{0} x^{\frac{m-1}{2}}, \theta=2, \varphi=1 \text { at } y=0
$$




$$
\frac{\partial \psi}{\partial y} \rightarrow 0, \theta \rightarrow 0, \varphi \rightarrow 0 \text { as } y \rightarrow \infty
$$

where $A=b\left(T_{w}-T_{\infty}\right)$ and $A_{1}=b\left(C_{w}-C_{\infty}\right)$.

\section{Lie Group Transformations}

The simplified form of Lie group transformations namely, the scaling group of transformations [8],

$$
x^{*}=x e^{\varepsilon c_{1}}, y^{*}=y e^{\varepsilon c_{2}}, u^{*}=u e^{\varepsilon c_{3}}, v^{*}=v e^{\varepsilon c_{4}}, \psi^{*}=\psi e^{\varepsilon c_{5}}, \theta^{*}=\theta e^{\varepsilon c_{6}}, \varphi^{*}=\varphi e^{\varepsilon c_{7}}
$$

Eqn. (14) may be considered as point transformation which transform coordinates $(x, y, u, v, \psi, \theta, \phi)$ to the

Coordinates $\left(x^{*}, y, u^{*}, v^{*}, \psi^{*}, \theta^{*}, \phi^{*}\right)$.

Substituting eqn. (14) in eqns. (10)-(13) we get,

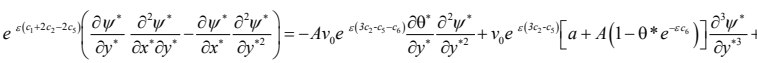

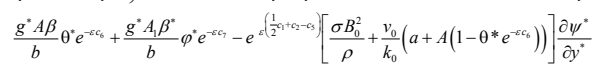

$$
\begin{aligned}
& \left.e^{\varepsilon\left(c_{1}+c_{2}-c_{s}-c_{0}\right)}\left(\frac{\partial \psi^{*}}{\partial y^{*}} \frac{\partial \theta^{*}}{\partial x^{*}}-\frac{\partial \psi^{*}}{\partial x^{*}} \frac{\partial \theta^{*}}{\partial y^{*}}\right)=e^{\varepsilon\left(2 c_{2}-c_{6}\right.}\right)\left(\frac{\kappa}{\rho c_{p}} \frac{\partial^{2} \theta^{*}}{\partial y^{* 2}}+\frac{16 \sigma^{*} T_{\infty}^{3}}{3 k^{*} \rho c_{p}} \frac{\partial^{2} \theta^{*}}{\partial y^{2}}\right)+\frac{Q_{1}}{\rho c_{p}} e^{-\varepsilon c_{c_{0}} \theta^{*}+} \\
& \frac{v_{0}}{\left(T_{w}-T_{\infty}\right) c_{p}} e^{\varepsilon\left(\epsilon_{2}-2 c_{c}\right)}\left(a+A\left(1-\theta^{*} e^{-\varepsilon c_{6}}\right)\right)\left(\frac{\partial^{2} \psi^{*}}{\partial y^{* 2}}\right)^{2} \\
& e^{\varepsilon\left(c_{1}+c_{2}-c_{5}-c_{7}\right)}\left(\frac{\partial \psi^{*}}{\partial y^{*}} \frac{\partial \varphi^{*}}{\partial x^{*}}-\frac{\partial \psi^{*}}{\partial x^{*}} \frac{\partial \varphi^{*}}{\partial y^{*}}\right)=D e^{\varepsilon\left(2 c_{2}-c_{7}\right)} \frac{\partial^{2} \varphi^{*}}{\partial y^{* 2}} k_{1} e^{-\varepsilon c_{7}} \varphi^{*}
\end{aligned}
$$

and

$$
\begin{aligned}
& e^{\varepsilon\left(c_{2}-c_{5}\right)} \frac{\partial \psi^{*}}{\partial y^{*}}=c x^{* m} e^{-\varepsilon c_{1} m}, e^{\varepsilon\left(c_{2}-c_{s}\right)} \frac{\partial \psi^{*}}{\partial y^{*}}=V_{0} x^{* \frac{m-1}{2}} e-^{\varepsilon\left(\frac{m-1}{2}\right) c_{1}}, \theta^{*}=1, \varphi^{*}=1 \text { at } y^{*}=0 \\
& \frac{\partial \psi^{*}}{\partial y^{*}} \rightarrow 0, \theta^{*} \rightarrow 0, \varphi^{*} \rightarrow 0 \text { as } y^{*} \rightarrow \infty
\end{aligned}
$$

The system will remain invariant under the group of transformations, we have

$$
\begin{aligned}
& c_{1}+2 c_{2}-2 c_{5}=3 c_{2}-c_{5}-c_{6}=3 c_{2}-c_{5}=-c_{6}=-c_{7}=\frac{1}{2} c_{1}+c_{2}-c_{5}, \\
& c_{1}+c_{2}-c_{5}-c_{6}=2 c_{2}-c_{6}=-c_{6}=4 c_{2}-2 c_{5}=4 c_{2}-2 c_{5}-4 c_{2}-2 c_{5}-c_{6}, \\
& c_{1}+c_{2}-c_{5}-c_{7}=2 c_{2}-c_{7}=-c_{7}, \\
& c_{2}-c_{5}=-c_{1} m \text { and } c_{1}-c_{5}=-c_{1}\left(\frac{m-1}{2}\right) .
\end{aligned}
$$

In this study we take $m=\frac{1}{2}$ and $c=1$, we get:

$$
c_{2}=\frac{c_{1}}{4}, c_{3}=\frac{c_{1}}{2}, c_{4}=\frac{c_{1}}{4}, c_{5}=\frac{3 c_{1}}{4}, c_{6}=0 \text { and } c_{7}=0
$$

In view of these, the boundary conditions become

$$
\begin{aligned}
& \frac{\partial \psi^{*}}{\partial y^{*}}=x^{* \frac{1}{2}}, \frac{\partial \psi^{*}}{\partial x^{*}}=V_{0} x^{* \frac{-1}{4}}, \theta^{*}=1, \varphi^{*}=1 \text { at } y^{*}=0 \\
& \frac{\partial \psi^{*}}{\partial y^{*}} \rightarrow 0, \theta^{*} \rightarrow 0, \varphi^{*} \rightarrow 0 \text { as } y^{*} \rightarrow \infty .
\end{aligned}
$$

We also assume volumetric heat source/sink rate $Q_{1}=Q_{0} x^{*-1 / 2}$ and the thermal conductivity $k_{1}=\Omega x^{*-1 / 2}$. The translation transformation in powers of $\varepsilon$ and keeping terms up to the order $\varepsilon$, we have

$$
\begin{aligned}
& x^{*}=x+\varepsilon x c_{1}, y^{*}=y+\frac{1}{4} \varepsilon y c_{1}, u^{*}=u+\frac{1}{2} \varepsilon u c_{1}, \\
& \psi^{*}=\psi+\frac{3}{4} \varepsilon \psi c_{1}, \theta^{*}=\theta, \varphi^{*}=\varphi .
\end{aligned}
$$

In terms of differentials these yield

$$
\frac{d x}{x c_{1}}=\frac{d y}{\frac{1}{4} y c_{1}}=\frac{d u}{\frac{1}{2} u c_{1}}=-\frac{d v}{\frac{1}{4} v c_{1}}=\frac{d \psi}{\frac{3}{4} \psi c_{1}}=\frac{d \theta}{0}=\frac{d \varphi}{0}
$$

Solving (21), we get

$$
\psi^{*}=x^{* 3 / 4} f(\eta), \quad \theta^{*}=g(\eta), \varphi^{*}=h(\eta), \quad \eta=y^{*} x^{*-1 / 4}
$$

Substituting (22) into (15-17) and (20), we get

$$
\begin{aligned}
& 4\left[\delta_{2}+\delta_{1}(1-g)\right] f^{\prime \prime \prime}-4 \delta_{1} g^{\prime} f^{\prime \prime}-2 f^{\prime 2}+3 f f^{\prime \prime}+4 \delta_{1} \alpha_{1} g+4 \delta_{3} \alpha_{2} h-4 \\
& {\left[M+S\left(\delta_{2}+\delta_{1}(1-g)\right)\right] f^{\prime}=0,} \\
& 4(3 R+4) g^{\prime \prime}+3 R P_{r}\left(3 f g^{\prime}+4 \Gamma g\right)+3 R P_{r} E_{c}\left(\delta_{2}+\delta_{1}(1-g)\right) f^{\prime \prime}=0, \\
& 4 h^{\prime \prime}+L_{e}\left(3 f h^{\prime}-4 \gamma h\right)=0, \\
& \text { with: }
\end{aligned}
$$

$f(0)=\lambda, f^{\prime}(0)=1, g(0)=1, h(0)=1, f^{\prime}(\infty) \rightarrow 0, g(\infty) \rightarrow 0, h(\infty) \rightarrow 0$

Here $\lambda$ is the suction $(\lambda>0)$ and it is injection $(\lambda<0)$.

\section{Skin-Friction Coefficient, Nusselt Number and Sher- wood Number}

The parameters of engineering interest for the present problem are the skin-friction coefficient, Nusselt number and the Sherwood number which indicate the physical wall shear stress, rate of heat transfer and rate of mass transfer, respectively. Which are defined as:

$$
C_{f}=\tau_{w} / \rho U^{1 / 2}, \quad N_{u}=U^{1 / 2} \rho c_{p} q_{w} / \mathrm{\kappa}\left(T_{w}-T_{\infty}\right) \text { and } S n=U^{1 / 2} J_{w} / D\left(C_{w}-C_{\infty}\right)
$$

Where $\left(\tau_{w}\right)$ is the shear stress along the stretching sheet, $\left(q_{w}\right)$ is the heat flux from the sheet and the mass flux $\left(J_{w}\right)$ from the sheet and those are defined as

$$
\tau_{w}=\mu(T)\left(\frac{\partial u}{\partial y}\right)_{y=0}, \quad q_{w}=-\frac{\kappa}{\rho c p}\left(\frac{\partial T}{\partial y}\right)_{y=0} \text { and } J_{w}=-D\left(\frac{\partial C}{\partial y}\right)_{y=0}
$$

Hence, skin-friction coefficient $C_{f}$, the Nusselt numbers $N_{u}$ and the Sherwood number $S_{n}$ as follows:

$$
C_{f}=\left(\delta_{2}+\delta_{1}(1-g(0))\right) f^{\prime \prime}(0), N_{u}=-g^{\prime}(0) \text { and } S_{n}=-h^{\prime}(0)
$$

\section{Result and Discussion}

Eqns. (23)-(26) are coupled nonlinear boundary value problems, these equations are solved numerically by fourth order mono-implicit Runge-Kutta method. It is difficult to study the influence of all parameters involved in the present problem.

In Figures 1-4(a, b and c), respectively; we have found that velocity profile increases, while temperature and concentration profiles decrease with the increase of each of fluid viscosity of temperature, fluid viscosity of concentration, temperature buoyancy and concentration buoyancy parameters. In absence of both magnetic field and porosity parameters effects on velocity, temperature and concentration which are illustrated in Figures 5 and 6 ( $\mathrm{a}, \mathrm{b}$ and $\mathrm{c}$ ), respectively; the velocity profile decreases, but the temperature and the concentration profiles increase with the increase of each of magnetic field and porosity parameters, and this is due to the fact that the thermal boundary layer increases with magnetic field parameter and porosity parameter.

The effects of the heat generation $(\Gamma>0)$ and the heat absorption $(\Gamma<0)$ on velocity, temperature and concentration profiles in Figure $7 \mathrm{a}-7 \mathrm{c}$, respectively. It is noticed that the velocity and the temperature 

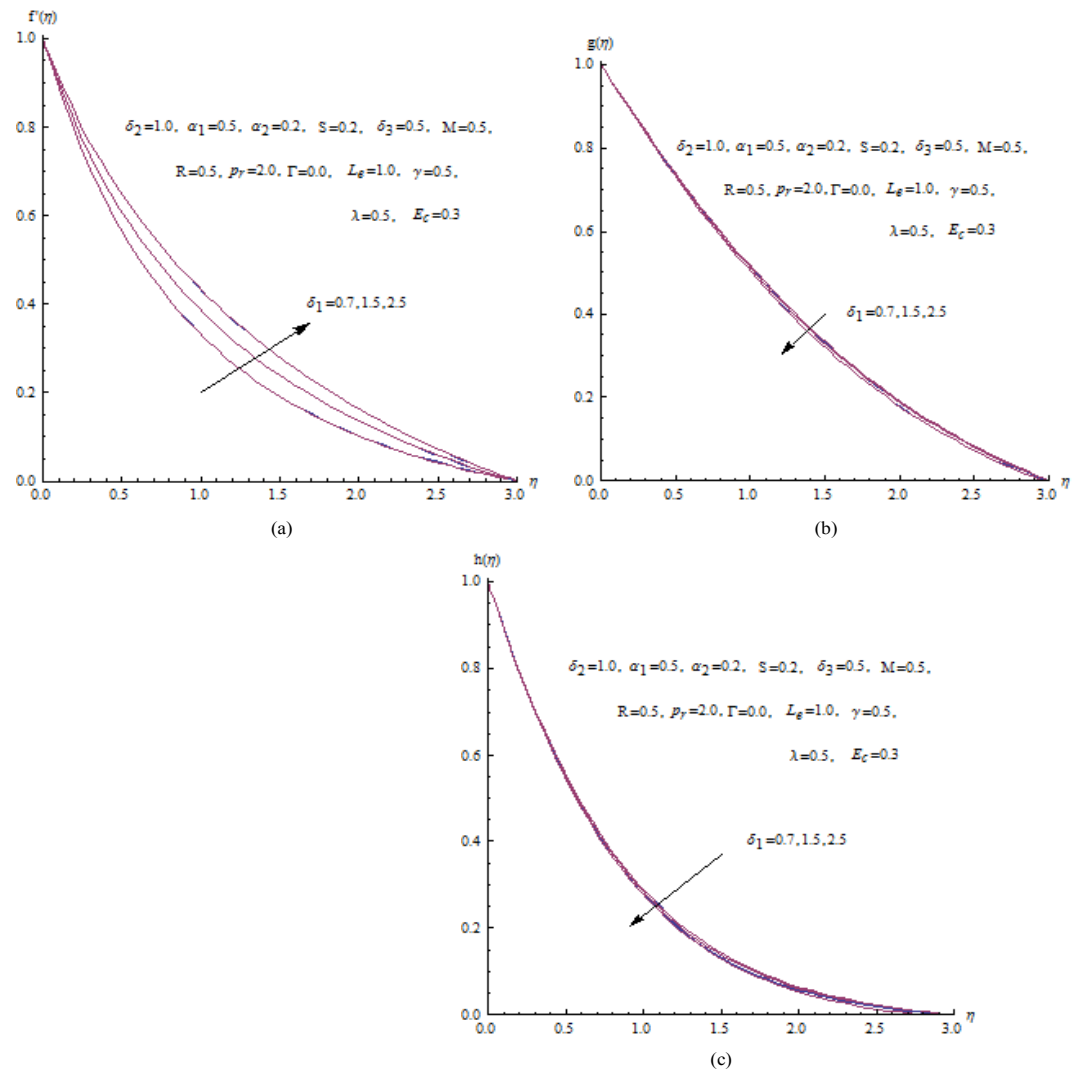

Figure 1: Influence of the fluid viscosity of temperature parameter on (a) velocity, (b) temperature and (c) concentration profiles.

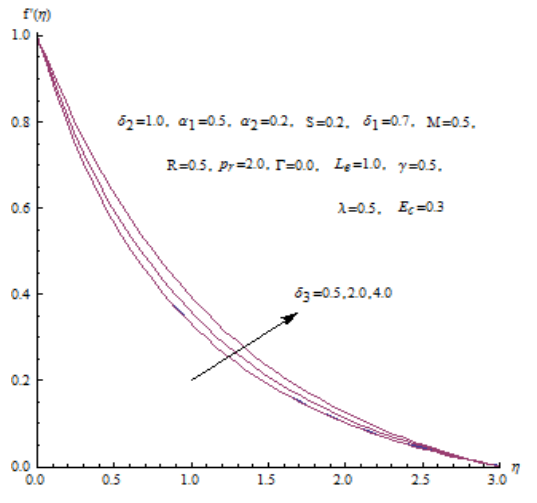

(a)

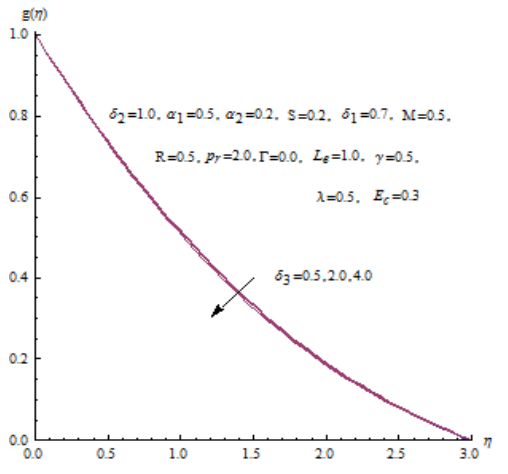

(b)

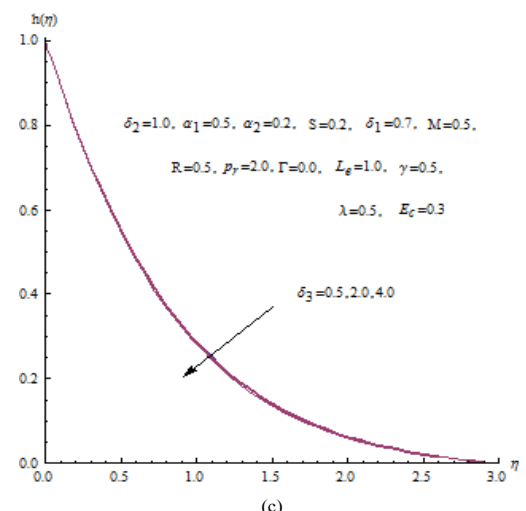

Figure 2: Influence of the fluid viscosity of concentration parameter on (a) velocity, (b) temperature and (c) concentration profiles. 

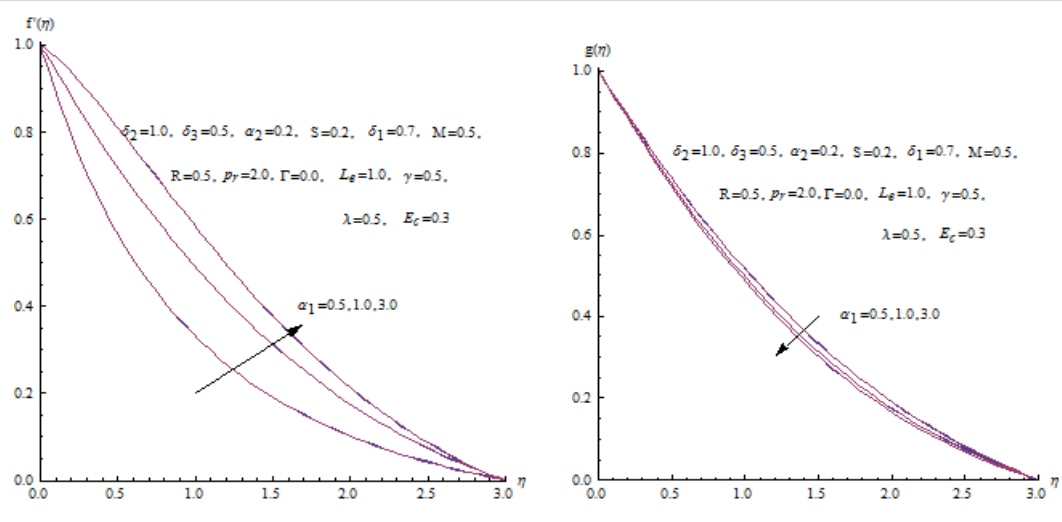

(a)

(b)

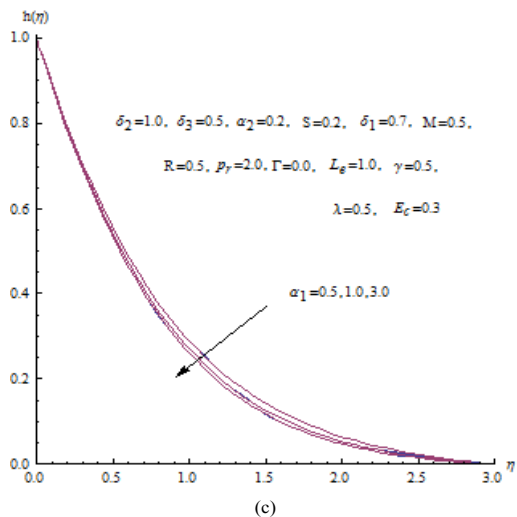

Figure 3: Influence of the temperature buoyancy parameter on (a) velocity, (b) temperature and (c) concentration profiles.

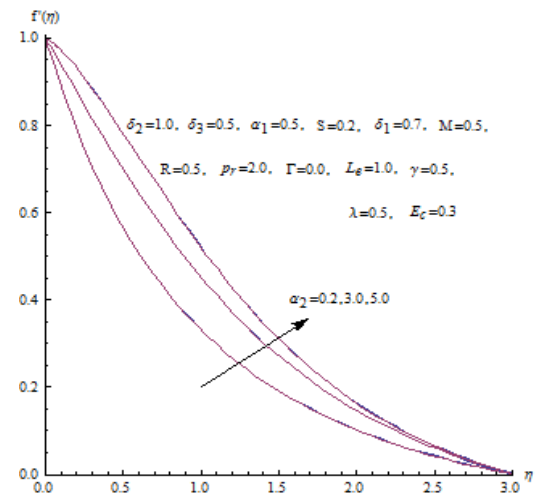

(a)

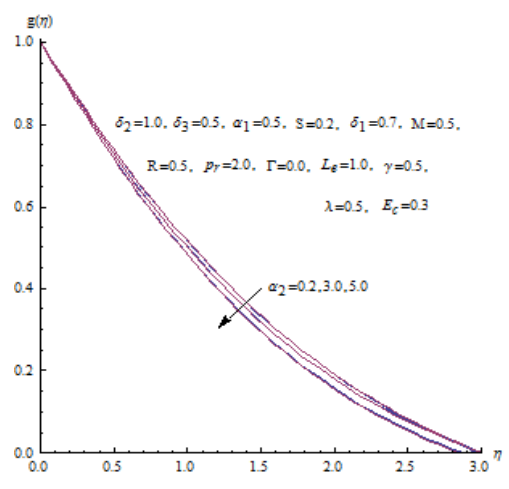

(b)

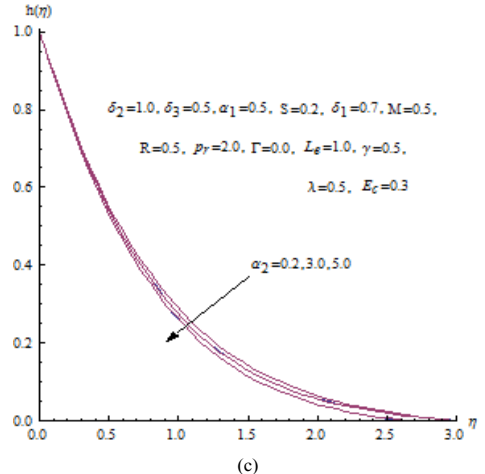

Figure 4: Influence of the concentration buoyancy parameter on (a) velocity, (b) temperature and (c) concentration profiles. 


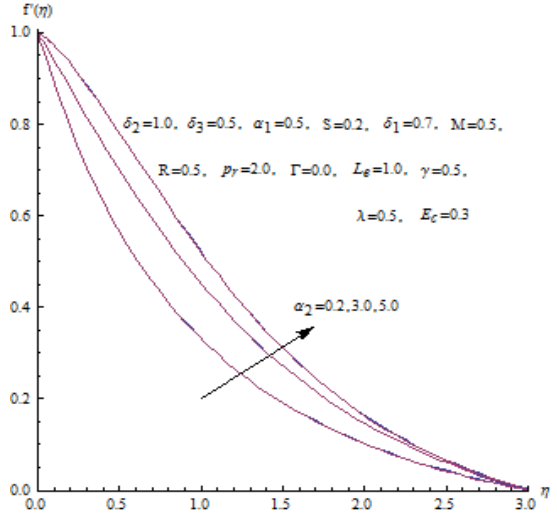

(a)

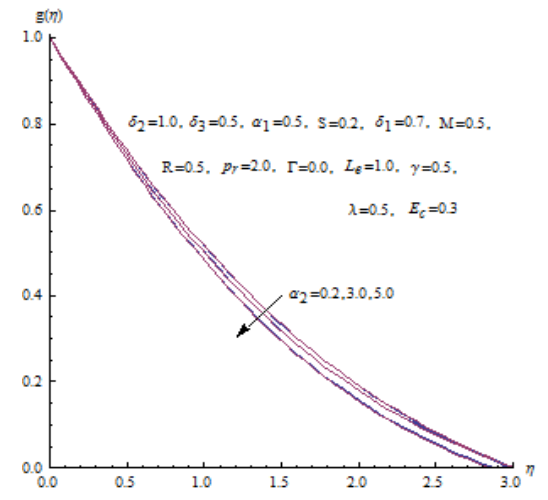

(b)

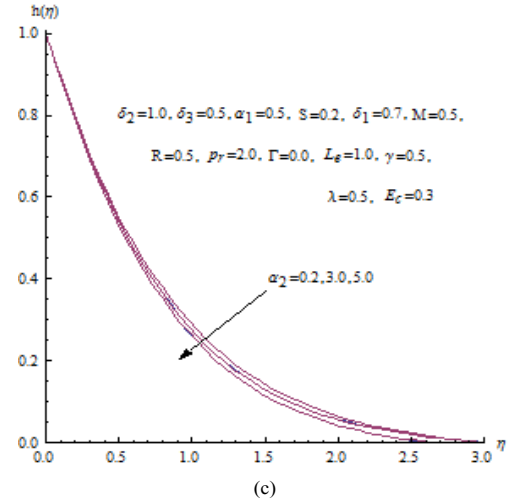

Figure 5: Influence of the magnetic field parameter on (a) velocity, (b) temperature and (c) concentration profiles.

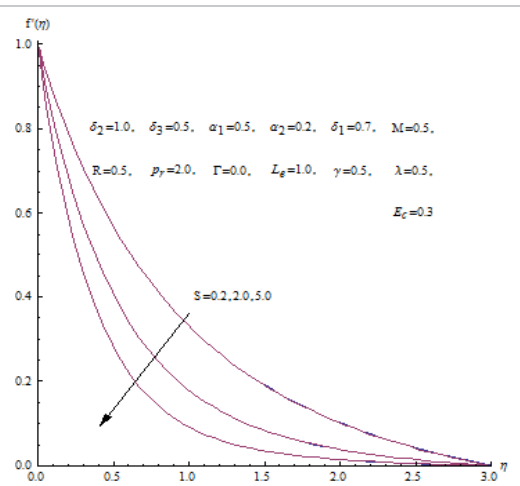

(a)

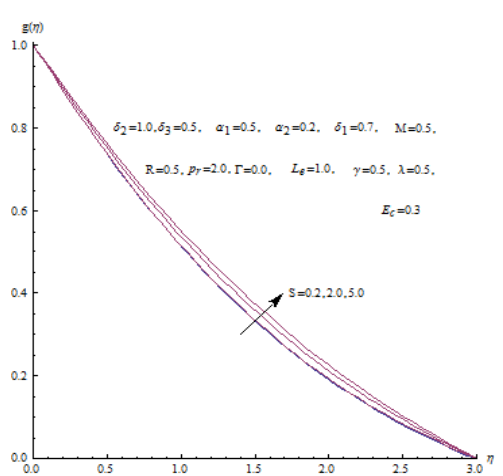

(b)

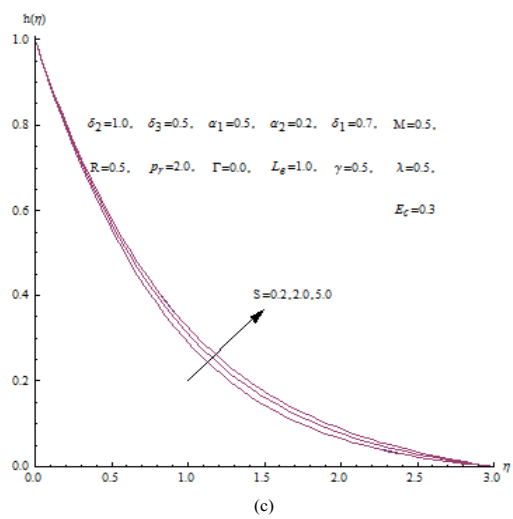

Figure 6: Influence of the porosity parameter on (a) velocity, (b) temperature and (c) concentration profiles. 


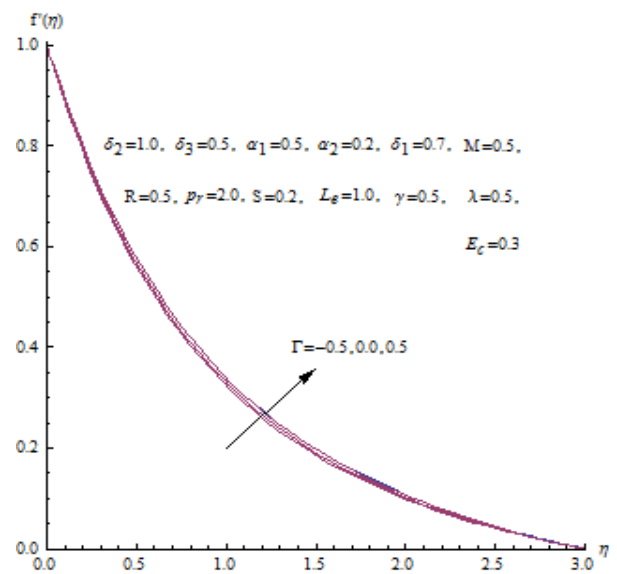

(a)

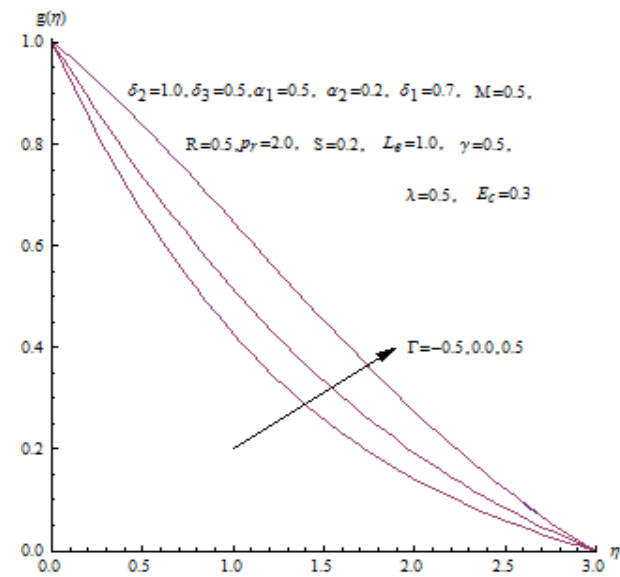

(b)

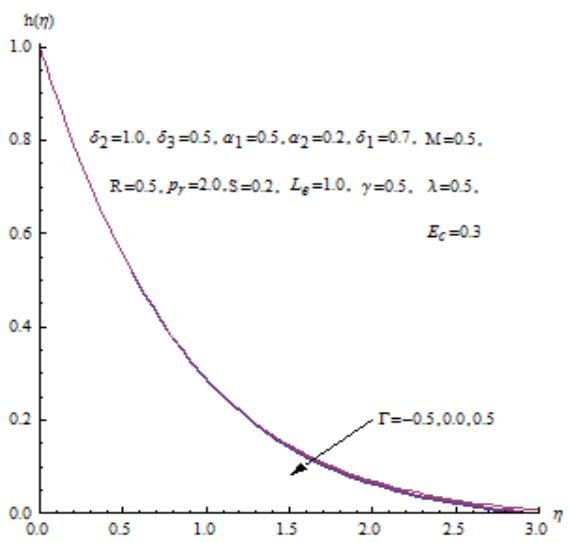

(c)

Figure 7: Influence of the heat generation/absorption parameter on (a) velocity, (b) temperature and (c) concentration profiles.

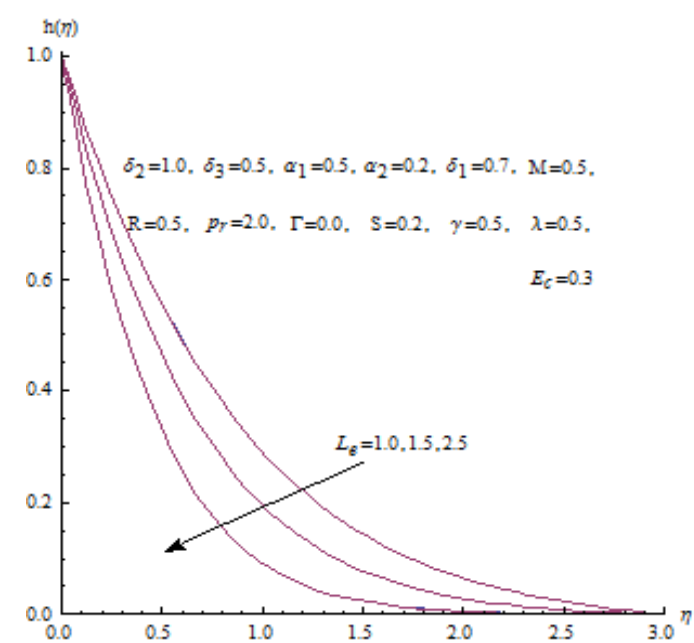

Figure 8: Influence of the Liwes number on concentration profile.

profiles increase, while the concentration profile decreases with increases of heat generation/absorption. The concentration profile decreases with increase of each of Liwes number and chemical reaction parameter in Figures 8 and 9, respectively. In Figure 10, the temperature profile is an increasing function of the increasing Eckert number. In

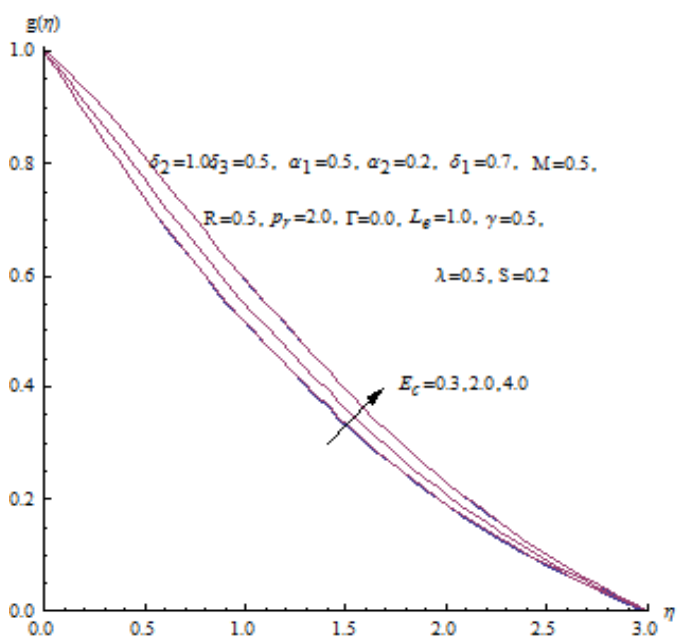

Figure 9: Influence of the chemical reaction parameter on concentration profile.

addition, at the sheet, the temperature also is different because of the existence of convection heat.

From Table 1, we found that, the skin-friction coefficient, Nusselt number and Sherwood Number increase with the increase of each 


\begin{tabular}{|c|c|c|c|c|c|c|c|c|c|c|c|c|}
\hline$\delta_{1}$ & $\delta_{3}$ & $\alpha_{1}$ & $\alpha_{2}$ & $M$ & $S$ & $\Gamma$ & $L_{\mathrm{e}}$ & $E_{c}$ & $\gamma$ & $C_{f}$ & $N_{u}$ & $S_{n}$ \\
\hline 0.7 & & & & & & & & & & -1.1885 & 0.548 & 1.111 \\
\hline 1.5 & & & & & & & & & & -1.0789 & 0.5567 & 1.1199 \\
\hline \multirow[t]{28}{*}{2.5} & & & & & & & & & & -0.96 & 0.5666 & 1.1312 \\
\hline & 0.5 & & & & & & & & & -1.1885 & 0.548 & 1.111 \\
\hline & 2 & & & & & & & & & -1.0601 & 0.555 & 1.1169 \\
\hline & 4 & & & & & & & & & -0.8916 & 0.5637 & 1.1244 \\
\hline & & 0.5 & & & & & & & & -1.1885 & 0.548 & 1.111 \\
\hline & & 2 & & & & & & & & -0.6116 & 0.5833 & 1.1412 \\
\hline & & 3 & & & & & & & & -0.2487 & 0.6013 & 1.1586 \\
\hline & & & 0.5 & & & & & & & -1.1885 & 0.548 & 1.111 \\
\hline & & & 3 & & & & & & & -0.6027 & 0.5767 & 1.1365 \\
\hline & & & 5 & & & & & & & -0.2176 & 0.6039 & 1.158 \\
\hline & & & & 0.5 & & & & & & -1.1885 & 0.548 & 1.111 \\
\hline & & & & 2 & & & & & & -1.7501 & 0.5157 & 1.0859 \\
\hline & & & & 4 & & & & & & -2.298 & 0.4916 & 1.0658 \\
\hline & & & & & 0.2 & & & & & -1.1885 & 0.548 & 1.111 \\
\hline & & & & & 2 & & & & & -1.8988 & 0.5053 & 1.0784 \\
\hline & & & & & 5 & & & & & -2.6598 & 0.4691 & 1.0534 \\
\hline & & & & & & -0.5 & & & & -1.2282 & 0.758 & 1.1098 \\
\hline & & & & & & 0 & & & & -1.1885 & 0.548 & 1.111 \\
\hline & & & & & & 0.5 & & & & -1.1311 & 0.2709 & 1.1112 \\
\hline & & & & & & & 1 & & & -1.1885 & 0.548 & 1.111 \\
\hline & & & & & & & 1.5 & & & -1.1947 & 0.5473 & 1.4395 \\
\hline & & & & & & & 2.5 & & & -1.2017 & 0.5468 & 2.0288 \\
\hline & & & & & & & & 0.3 & & -1.1885 & 0.548 & 1.111 \\
\hline & & & & & & & & 2 & & -1.1711 & 0.4218 & 1.1114 \\
\hline & & & & & & & & 4 & & -1.1578 & 0.2718 & 1.1116 \\
\hline & & & & & & & & & 0.5 & -1.1885 & 0.548 & 1.111 \\
\hline & & & & & & & & & 1.5 & -1.1964 & 0.5471 & 1.5482 \\
\hline & & & & & & & & & 2.5 & -1.1993 & 0.5463 & 1.8778 \\
\hline
\end{tabular}

Table 1: Numerical of the Skin-friction coefficient, Nusselt number and Sherwood Number for different $\delta_{1}, \delta_{3}, \alpha_{1}, \alpha_{2}, \Gamma, M, S, L_{e}, E_{c}$ and $\gamma$.

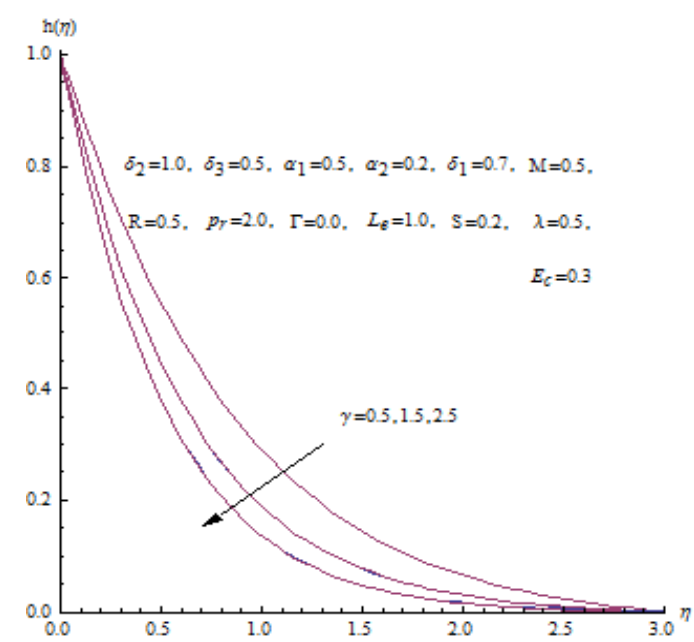

Figure 10: Influence of the Eckert number on temperature profile.

of fluid viscosity of temperature, fluid viscosity of concentration, temperature buoyancy and concentration buoyancy parameters. The skin-friction coefficient, Nusselt number and Sherwood Number decrease with the increase of each of the magnetic field and the porosity parameters.

The skin-friction coefficient and Sherwood Number increase while Nusselt number decreases with the increase of each of heat generation/ absorption and Eckert number. And in case of increasing of each of the Liwes number and chemical reaction parameter, it is observed that skin-friction coefficient and Nusselt number decrease but Sherwood Number increases (Table 1 and Figures 1-7).

\section{Conclusions}

In the present study, Magnetohydrodynamic and chemical reaction effects of variable viscosity on heat generation and mass transfer viscous dissipation fluid in the presence of suction/injection through porosity is solved numerically. It is observed that:

(1) The velocity profile, the skin-friction coefficient, Nusselt number and Sherwood Number increase while the temperature and concentration profiles decrease with the increase of each of fluid viscosity of concentration, temperature buoyancy and concentration buoyancy parameters (Figures 8-10).

(2) The velocity profile, the skin-friction coefficient, Nusselt number and Sherwood Number decrease, but the temperature and the concentration profiles increase with the increase of each of magnetic field and porosity parameters.

(3) The velocity, the temperature profiles, the skin-friction coefficient and Sherwood Number increase, while the concentration profile and Nusselt number decrease with increases of heat generation/ absorption.

(4) The concentration profile, the skin-friction coefficient and Nusselt number decrease but Sherwood 
Citation: Abdel-Rahman GM, Alessa NA (2018) Lie Group for MHD and Reaction Porosity Effects of Variable Viscosity on Heat Generation and Mass Transfer Fluid. J Generalized Lie Theory Appl 12: 287. doi: 10.4172/1736-4337.1000287

Number increases with increase of each of Liwes number and chemical reaction.

(5) The temperature profile, the skin-friction coefficient and Sherwood Number increase but Nusselt number decreases with increase of Eckert number.

\section{References}

1. Bluman GW, Kumei S (1989) Symmetries and Differential Equations. Springer New York.

2. Olver J (1989) Application of Lie Groups to Differential Equations. Springer, New York.

3. Boutros YZ, Abd-el-Malek MB, Badran NA, Hassan HS (2007) Lie-group method solution for two- dimensional viscous flow between slowly expanding or contracting walls with weak permeability. Appl. Math Model 31: 1092-1108

4. Andersson IHI, Aarseth JB, Dandapat BS (2000) Heat transfer in a liquid film on an unsteady stretching surface. International Journal of Heat and Mass Transfer 43: 69-74

5. Saikrishnan P, Roy S (2003) Non-Uniform Slot Injection (Suction) into Steady Laminar Water Boundary Layers over (i) a Cylinder and (ii) a Sphere. International Journal of Engineering Science 41: 1351-1365.
6. Roy S, Saikrishnan P (2003) Non-Uniform Slot Injection (Suction) into Steady Laminar Water Boundary Layer Flow over a Rotating Sphere. International Journal of Heat and Mass Transfer 46: 3389-3396.

7. Elbashbeshy EMA, Bazid MAA (2004) Heat Transfer in a Porous Medium ove a Stretching Surface with Internal Heat Generation and Suction or Injection. Applied Mathematics and Computation 158: 799-807.

8. Mukhopadhyay S, Layek GC, Samad SA (2005) Study of MHD Boundary Layer Flow over a Heated Stretching Sheet with Variable Viscosity. International Journal of Numerical Methods for Heat and Fluid Flow 48: 4460-4466.

9. Mukhopadhyay S, Layek GC (2008) Effects of Thermal Radiation and Variable Fluid Viscosity on Free Convective Flow and Heat Transfer Past a Porous Stretching Surface. International Journal of Heat and Mass Transfer 51: 2167-2178.

10. Loganathan P, Arasu PP (2010) Lie group analysis for the effects of variable fluid viscosity and thermal radiation on free convective heat and mass transfer with variable stream condition. Engineering 2: 625-634.

11. Batchelor GK (1987) An Introduction to Fluid Dynamics. Cambridge University Press, London.

12. Rashed GMA (2016) Chemical Entropy Generation and MHD Effects on the Unsteady Heat and Fluid Flow through a Porous Medium. J of Applied Mathematics, pp: 1-9. 